\title{
Operation-Aware Health Management for Environmental Control and Life Support System
}

\author{
Shijie Tang \\ University of South Carolina, Columbia, South Carolina, 29208 \\ shijie@email.sc.edu
}

\begin{abstract}
To enable effective management, planning, and operations for future missions that involve a crewed space habitat, operational support must be migrated from Earth to the habitat. Intelligent System Health Management technologies (ISHM) promise to enable the future space habitats to increase the safety and mission success while minimizing operational risks. Aligning with this objective, my Ph.D. study aims to develop fault diagnosis, prognosis, and automatic contingency management strategies for Environmental Control and Life Support Systems (ECLSS). Innovations of the research include a combination of physics-based and data-driven modeling, low-cost diagnosis and prognosis, probabilistic reasoning, and optimization for decisionmaking. Data from Photovoltaic (PV) arrays and Water Recycling System (WRS) deployed at NASA Ames Research Center's Sustainability Base will be used for comparison studies with existing methods and technology validation and verification of the proposed system.
\end{abstract}

\section{PROBLEM STATEMENT}

Most of the planning and management of space operations are conducted locally on earth, limiting realtime input from crew members during space missions. Space habitat crew personnel can be given the opportunity to manage, plan and operate much of the purpose themselves, by migrating operational support from Earth to the habitat in space. This task will require significant automation and decision support software, which will benefit a small sized crew, by enabling new monitoring, tracking, and management capabilities onboard the habitat and related NASA platforms.

Advances in intelligent health management technologies increase mission safety and success while minimizing the operational risks and costs. This innovation is essential to future habitats stationed on other planets, asteroids, or lunar surfaces.

The ECLSS's reliability and survivability are critical to NASA, especially in long-term human space exploration missions. The Health Management of life support systems consists of several components, among which power, waste processing, water recovery, and

Shijie Tang et al. This is an open-access article distributed under the terms of the Creative Commons Attribution 3.0 United States License, which permits unrestricted use, distribution, and reproduction in any medium, provided the original author and source are credited. biomass processing systems, etc. are of primary importance. The ECLSS is considerable in size and a complicated generation (water, oxygen, food) and distribution (waste, CO2, N2) system. Due to the crew's critical dependence on such a complex system, the health management of ECLSS becomes crucial to NASA's mission success rates.

Maintaining the proper functionalities of ECLSS, under nominal (healthy) and faulty (some components or subsystems degrade or fail to work) conditions, is the central goal of health management. The underlying issues are how to model, identify, analyze, predict, and synthesize new system conditions for NASA's ECLSS in dynamic situations in real time. These conditions consist of both complex nonlinear continuous and discrete dynamics. Continuous dynamics include mathematical models of a network composed of batteries, loads, continuous controllers, valves, pressure regulators, and process control units. Discrete dynamics can be defined by a discrete finite automation that models various discrete events, for example, a binary list of system states. Therefore, the ECLSS can be modeled as a hybrid system and can be monitored and controlled by a combination of supervisory and distributed health management systems. In such a system, the distributed system locally deals with high bandwidth signals, while the supervisory system deals with processed data from the distributed system. As a result, the required bandwidth of the overall ECLSS is low. Moreover, the supervisory-level system provides an optimization overall system reconfiguration and planning. Finally, an architecture that permits rapid fault, leakage, and component malfunction detection, as well as, fault mitigation, and reconfiguration based on mission requirements is required.

Although this design approach has significant advantages, it brings some immediate challenges. The first one is that the diagnosis and prognosis functionalities must be distributed to local components. These local components are often supported by microprocessors and embedded systems, which have limited computing, storage, and communication capabilities. They cannot support complicated diagnosis and prognosis algorithms. Therefore, computationally effective diagnosis and prognosis algorithms need to be 
developed. Second, information fusion from health condition of the distributed components to a systemlevel health condition and its optimal fault mitigation strategy remains an open problem and needs to be addressed.

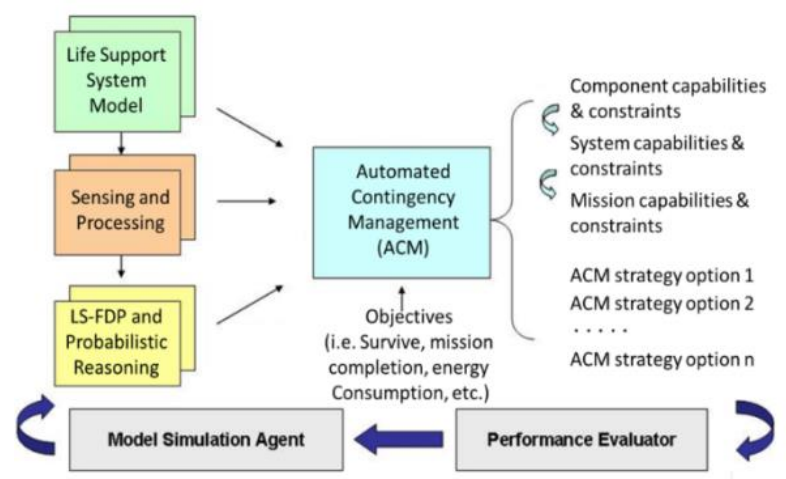

Fig. 1. Dynamic ACM Interfaces and Proposed Modules

\section{EXPECTED CONTRIBUTIONS}

My Ph.D. research has a structure shown in Fig. 1 and the contributions include:

1) Model the ECLSS based on physical dynamics and data from the Sustainability Base at Ames [1]. Simulate the WRS [2] and PV with fault injection of discrete and continuous fault modes.

2) Develop a hierarchical structure that enables to deal with multidimensional FDP in ECLSS with capabilities of FDP reasoning from the health condition of devices to subsystems, which are grouped devices to realize specific functions in the ECLSS. In this FDP design, different methods, such as extended Kalman filter [3], particle filter, and machine learning techniques will be used according to the nature of different fault modes. This function provides the system a health indicator by fusing information from components.

3) Develop an Automated Contingency Management (ACM) framework for ECLSS, Fig. 4. The ACM framework includes fault detection, fault isolation, Lebesgue sampling based extended Kalman filter (LS-EKF) based fault identification and Dynamic Programming (DP) for fault mitigation.

4) Integrate machine learning based diagnosis and prognosis approaches (deep belief network and convolutional neural networks) and a Matlab abnormal condition detection toolbox, ACCEPT from NASA [1], to analyze data from WRS and PV systems for operation awareness.

5) Develop a method to distinguish sensor fault and component fault for accurate contingency strategy implementation.

6) Verify and validate the proposed system with models under different operating condition and data from NASA’s Sustainability Base.

\section{RESEARCH PLAN}

This Ph.D. research consists of four tasks with details being described as follows.

\subsection{Work Performed}

The WRS, which collects condensate in the air, used water, and recycle them into water, is one of the critical subsystems in ECLSS. To accommodate faults in WRS, an ACM framework is developed, Fig. 3. Different fault modes, both discrete and continuous ones, can be injected. Faults are detected and estimated by a Lebesgue sampling based approach [3]. With fault state estimation; DP is used to optimize the energy consumption and maintain the WRS in a degraded but acceptable operating condition. Fig. 2 shows the preliminary results for a filter clogging fault and the optimal time to take action by increasing the output pressure of pump [4].

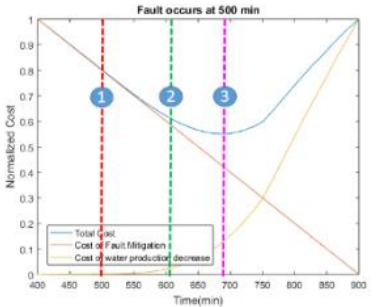

(a)

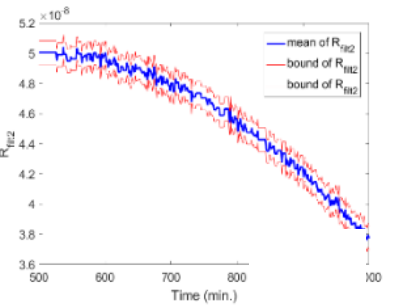

(b)
Fig. 2 (a) Optimization with fault at the $500^{\text {th }}$ min. (b) FDP results by LS-EKF.

A series of simulations on LS-EKF based FDP, discrete pump fault, continuous filter clogging faults, and dynamic programming (DP) based optimization had been conducted to demonstrate the effectiveness of the proposed method. The ACM strategy developed in this research is application agnostic and can be applied more generally to other subsystems, such as power supply, waste processing, and biomass processing and other NASA systems for outer space missions.

\subsection{Remaining Work}

1) Although we have preliminary results on WRS, the work needs to be expanded to full-scale ECLSS. Considering the time limitation, we will include PV system such that it simulates two interacting systems. Since the ACM system needs to integrate fault diagnosis and prognosis, reasoning on system performance from multi-dimensional interacting faults, and optimization, a hierarchical structure is developed, Fig. 3.

The hierarchical structure includes high-level ACM; middle-level distributed system health condition reasoning and low-level FDP. In my thesis, a Finite State Machine (FSM) will represent 
the high-level ACM. To efficiently optimize the operation and safety of ECLSS, the middle-level distributed reasoning is based on network structural modeling. It takes local FDP information and device observations and ECLSS scheduling to achieve an accurate and precise assessment of ECLSS health and operational risk. Moreover, it will study the effects of uncertainties of diagnosis and prognosis in hierarchical probabilistic reasoning. The lowlevel FDP will utilize a Lebesgue sampling based approach to enable real-time FDP on the local processors for distributed configuration.

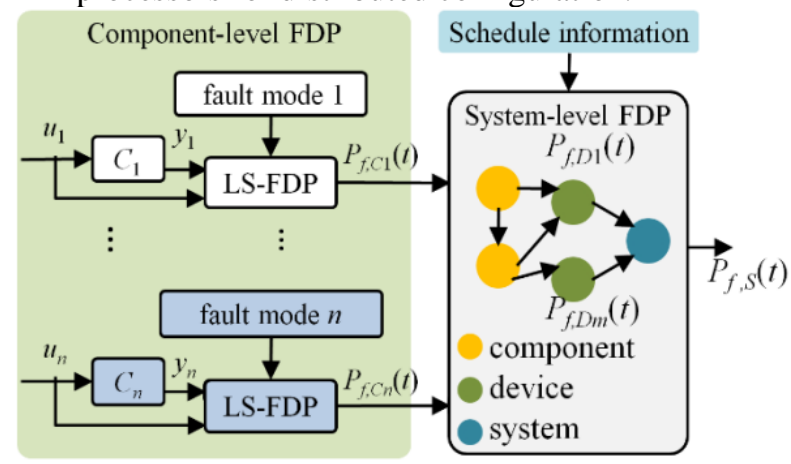

Fig. 3 Distributed Hierarchical FDP

2) ACM optimization must be addressed by considering the capabilities and constraints of the components and systems, which are indicated by the FDP information. Since the violation of any of the constraints is detrimental to the ACM solution validity, the impact of any constraint violation on the cost function must be negative. Apart from DP, my thesis will also study optimization based on genetic algorithm with consideration of human in the loop.

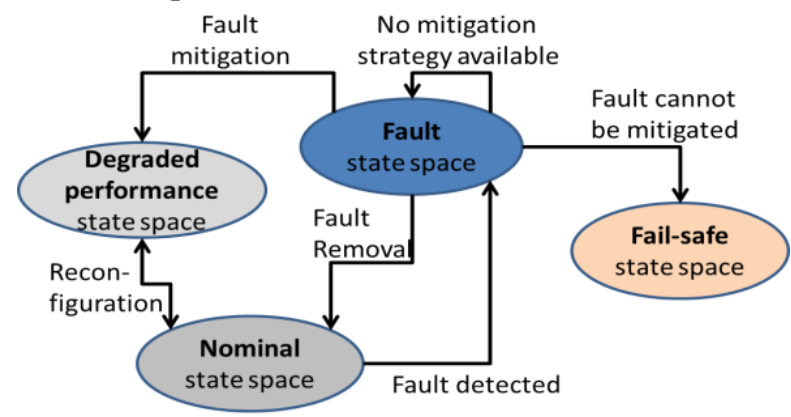

Fig. 4 ACM modeling

3) To address sensor failure, a model-based nonlinear fault diagnostic method will be studied. The method consists of a bank of nonlinear adaptive fault diagnostic estimators, which are compared with actual sensor signals to obtain a set of structured diagnostic residuals. Based on the residual response, the presence of sensor faults or component faults can be determined.
4) Verification and validation on NASA Sustainability Base. In my thesis research, I will use the publicly available data (under healthy and faulty operating conditions) and simulation data from high-fidelity models (with fault free and faults being injected) for system development and prototype. The available dataset that can be used includes PV data from NASA Sustainability Base and WRS data.

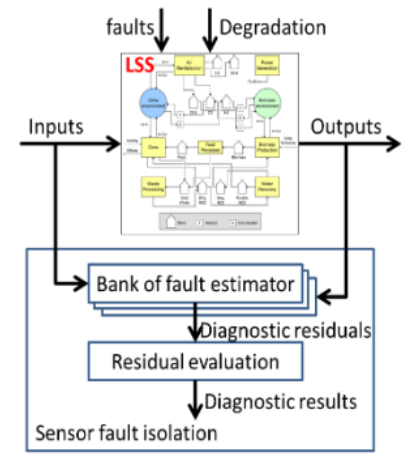

(a)

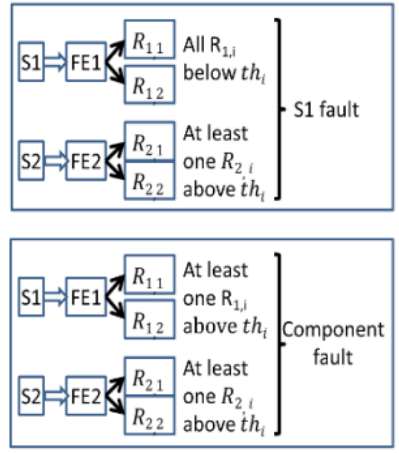

(b)
Fig. 5: (a) Sensor fault isolation scheme; (b) a 2-sensor case of sensor/component fault isolation

\section{CONCLUSION}

My thesis aims to develop an ACM framework for the life support system and use PV and WRS as a testbed for technical development and verification. The proposed system consists of hierarchical Bayesian probabilistic reasoning; crew activities involved optimization, Verification and validation on NASA Sustainability Base. With the development of this new methodology, I plan to complete my Ph.D. studies in the next three years with a focus on ECLSS simulation FDP, optimization, probabilistic reasoning, machine learning and system integration.

\section{REFERENCES}

[1] C. Poolla, A. Ishihara, S. Rosenberg, R. Martin, A. Fong, S. Ray, and C. Basu, "Neural network forecasting of solar power for NASA Ames sustainability base," IEEE Symp. Comput. Intell. Appl. Smart Grid, CIASG, 2015.

[2] I. Roychoudhury, V. Hafiychuk, and K. Goebel, "Modelbased diagnosis and prognosis of a water recycling system," Aerosp. Conf. 2013 IEEE, pp. 1-9, 2013.

[3] W. Yan, B. Zhang, "Extended Kalman filter in Lebesgue sampling framework with an application to Li-ion battery diagnosis and prognosis," 3rd European Conf. PHM (PHME), 2016, Bilbao, Spain.

[4] S. Tang, B. Zhang, W. Yan, A. Thakker, S. Vivanco, R. Martin, and C. Moore, "Operation-Aware ISHM for Environmental Control and Life Support in Deep Space Habitants," AIAA Inf. Syst. Infotech @ Aerosp., 2018. 